\title{
Computers can't give credit: How automatic attribution falls short in an online remixing community
}

\author{
Andrés Monroy-Hernández ${ }^{1,2}$, Benjamin Mako Hill ${ }^{1}$ \\ Jazmin Gonzalez-Rivero ${ }^{2}$, danah boyd ${ }^{2}$ \\ ${ }^{1}$ Massachusetts Institute of Technology, Cambridge, MA 02139 \\ ${ }^{2}$ Microsoft Research, Cambridge, MA 02142 \\ \{amonroy,mako\}@mit.edu $\{\mathrm{t}$-jazgo,dmb $\} @$ microsoft.com
}

\begin{abstract}
In this paper, we explore the role that attribution plays in shaping user reactions to content reuse, or remixing, in a large user-generated content community. We present two studies using data from the Scratch online community a social media platform where hundreds of thousands of young people share and remix animations and video games. First, we present a quantitative analysis that examines the effects of a technological design intervention introducing automated attribution of remixes on users' reactions to being remixed. We compare this analysis to a parallel examination of "manual" credit-giving. Second, we present a qualitative analysis of twelve in-depth, semi-structured, interviews with Scratch participants on the subject of remixing and attribution. Results from both studies suggest that automatic attribution done by technological systems (i.e., the listing of names of contributors) plays a role that is distinct from, and less valuable than, credit which may superficially involve identical information but takes on new meaning when it is given by a human remixer. We discuss the implications of these findings for the designers of online communities and social media platforms.
\end{abstract}

\section{Author Keywords}

remixing, attribution, credit-giving, user-generated content, online communities

\section{ACM Classification Keywords}

H.5.3 Group and Organization Interfaces

\section{General Terms \\ Design, Experimentation, Human Factors}

\section{INTRODUCTION}

Networked information technologies have changed the way people use and reuse creative - and frequently copyrighted materials. This change has generated excitement, and heated

Permission to make digital or hard copies of all or part of this work for personal or classroom use is granted without fee provided that copies are not made or distributed for profit or commercial advantage and that copies bear this notice and the full citation on the first page. To copy otherwise, or republish, to post on servers or to redistribute to lists, requires prior specific permission and/or a fee.

CHI 2011, May 7-12, 2011, Vancouver, BC, Canada.

Copyright 2011 ACM 978-1-4503-0267-8/11/05...\$10.00.

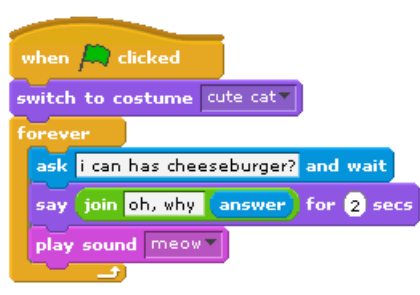

Figure 1. An example Scratch program written using programming "blocks".

debate, among content-creators, technologists, legal academics, and media scholars. Media theorist Lev Manovich argues that remixing is an ancient cultural tradition (e.g., he has suggested that ancient Rome was a "remix" of ancient Greece) but that information technologies have accelerated these processes and made remixing more salient [14]. Sinnreich et al. argue that "configurable culture" has been significantly transformed by networked technologies which introduce perfect copying and allow people not only to be inspired by extant creations but to remix the original works themselves [25]. Legal scholars have stressed the importance of remixing in cultural creation broadly and warned that current copyright and intellectual property laws may hinder creativity and innovation $[11,1]$.

Several of the most influential scholarly explorations of remixing as a cultural phenomenon have focused on youth's remixing practices. For example, work on remixing by Jenkins [10] and Ito [9] has focused on young people's use and re-use of media. Palfrey and Gasser have suggested that the cultural practices of "digital native" youth have had a significant transformative effect on our culture [17]. Throughout his book "Remix," Lessig uses youth's reuse practices to support an argument against what he considers excessive copyright legal protection [12].

Yet, despite a wide interest in remixing and authorship, researchers have only recently engaged in empirical research on the subject [4]. Several recent treatments have presented studies of video remixing communities [5, 24], music remixing communities [4], collaborative video game communities [13] and social network sites [18]. There is also another quantitative study of our empirical setting [8] focused on characterizing the variety of responses to remixing. These studies have tended to be general and largely descriptive ex- 
aminations of remixing practice. This work has pointed to the existence of norms [5] and the territoriality of digital creators [26] and has considered issues of motivation [4]. However, empirical work has yet to unpack in detail the key social mechanisms that scholars have suggested drive behavior, norms, and motivation in remixing communities.

Perhaps no mechanism has been more frequently cited as critical for remixing activity than attribution and the related phenomena of plagiarism, reputation, and status. For example, recent survey-based work has suggested that the "authenticity and legitimacy" of creative work "are premised on the explicit acknowledgment of the source materials or 'original creator"' and that such acknowledgment is a key component of how adults assess the fairness or ethical nature of content reuse [25]. Attribution, in this sense, can be seen as an important way that people distinguish remixing from "theft."

Judge and law professor Richard Posner stresses the importance of attribution and explains that this is important even when there is no monetary benefit to being attributed. For example, he explains that European copyright law is based on a doctrine of "moral rights" that "entitles a writer or other artist to be credited for his original work and this 'attribution right', as it is called, would give him a legal claim against a plagiarist." Posner also explains that "acknowledgment" of another's contributions to a derivative negates any charge of plagiarism, although it may not establish originality [20]. Attribution plays such an important role in remix culture that Creative Commons made a requirement for attribution a component of all their licenses after more than $97 \%$ of licensors opted to require attribution when it was offered as a choice [2].

Young people's perceptions of attribution and complications around copying have also been examined. An article by Friedman describes that adolescents who allowed "computer pirating" - the unauthorized copying of computer programs - did so because technological affordances made it difficult for adolescents to identify "harmful or unjust consequences of computer-mediated actions" [7]. In a second study, psychologists Olson and Shaw have found that by five years old, "children understand that others have ideas and dislike the copying of these ideas" [16].

Yet, despite the fact that researchers in human computer interaction have begun to explore the complexity of attribution and cited its importance to remixing [13], many designers of online communities pay little attention to issues of attribution in their designs - a fact that is reflected in user behavior. For example, research on the use of photos from the photo sharing site Flickr [22], as well as a number of other usergenerated content communities [23], suggests that most reusers fail to attribute re-used content in ways that public-use licenses require. Although theory and survey based work points to a need to design for attribution in user-generated content communities, we still know very little about how attribution works or how designers might go about doing so.
Indeed, our study suggests that the most obvious efforts to design for attribution are likely to be ineffective.

In this paper, we employ a mixed methods approach that combines qualitative and quantitative analyses to explore users' reactions to attribution and its absence in a large remixing community. First, we introduce our empirical setting; using qualitative data from users forums and comments, we present a rich description of remixing and evidence to support our core proposition that credit plays a central role in remixing in our environment. Second, we contextualize and describe a technological intervention in our setting, responding directly to several user suggestions, that automated the attribution of creators of antecedent projects when content was remixed. Third, we present a tentative quantitative analysis of the effect of this intervention along with a parallel analysis of the practice of manual creditgiving. We find that credit-giving, done manually, is associated with more positive reactions but that automatic attribution by the system is not associated with a similar effect. Fourth, we present analysis of a set of in-depth interviews with twelve users which helps confirm, and add nuance and depth to, our quantitative findings.

Our results suggest that young users see an important, if currently under-appreciated and under-theorized, difference between credit and attribution. Credit represents more than a public reference to an "upstream" user's contributions. Coming from another human, credit can involve an explicit acknowledgment, an expression of gratitude, and an expression of deference, in a way that simple attribution can not. Our results suggest that identical attribution information means something very different to users when it comes from a computer, and when it comes from a human - and that users often feel that acknowledgment is worth much less when it comes from a system. We conclude that designers should create affordances that make it easier for users to credit each other, rather than to merely pursue automated means of acknowledgment.

Our study offers two distinct contributions for social scientists and for technology designers. The first is an improved understanding of the way that attribution and credit work in user-generated content communities. The second is a broader contribution to the literature on design that suggests an important limitation to technologists' ability to support community norms and a suggestion for how designers might create affordances. Functionality that allows users to express information that a system might otherwise show automatically may play an important role in successful design for social media environments.

\section{SCRATCH: A COMMUNITY OF YOUNG REMIXERS}

The Scratch online community is a free and publicly available website where young people share their own video games, animated stories, interactive art, and simulations [15]. Participants use the Scratch programming environment [21], a desktop application, to create these interactive projects by putting together images, music and sounds with programming command blocks (See Figure 1). 


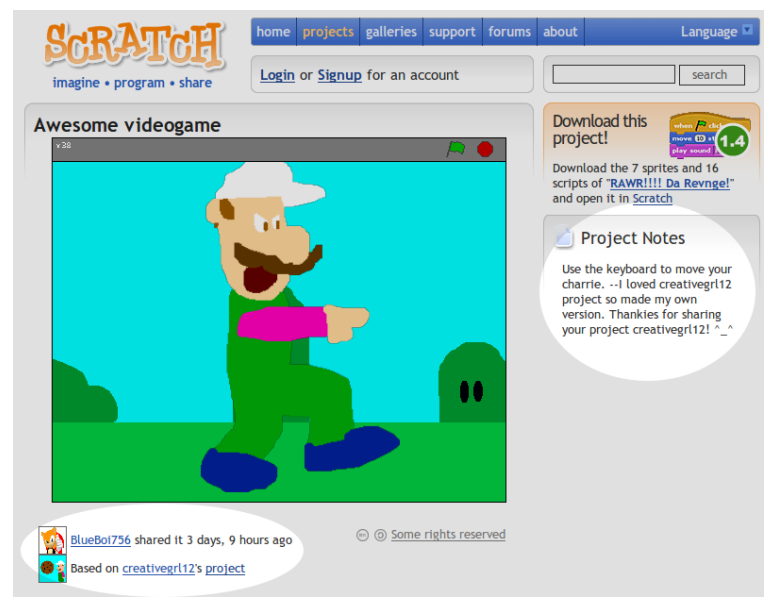

Figure 2. Screenshot of a Scratch remix project highlighting automatic (the area inside the bottom left circle) and manual (the area in the top right circle) attribution.

The Scratch website was officially announced in 2007 and, as of September 2010, has more than 600,000 user accounts who have shared 1.3 million projects. At the time of writing, Scratch users share on average one new project per minute. Examples of projects range from an interactive virtual cake maker, to a simulation of an operating system, to a Pokemon-inspired video game, to an animation about climate change, to tutorials on how to draw cartoons. Like other user-generated content websites, such as YouTube or Flickr, Scratch projects are displayed on a webpage (See Figure 2) where people can interact with them, read metadata and give feedback. Visitors can use their mouse and/or keyboard to control a video game or other type of interactive projects or simply observe an animation play out in a web browser. Metadata displayed next to projects includes a text-based description of the project, the creator's name, the number of views, downloads, "love its," remixes, and galleries (i.e., sets of projects) that the project belongs to. Users can interact with projects by giving feedback in the form of tags, comments, or clicks on the "love it" button, and can flag a project as "inappropriate" for review by site administrators.

Participants' self-reported ages range primarily from 8 to 17 years-old with 12 being the median. Thirty-six percent of users self-report as female. A large minority of users are from the United States (41\%) while other countries prominently represented include the United Kingdom, Thailand, Australia, Canada, Brazil, South Korea, Taiwan, Colombia and Mexico. About $28 \%$ of all users - more than 170,000 have uploaded at least one project.

\section{Remixing in Scratch}

Scratch users can download any project shared on the website, open it up in the Scratch authoring environment, learn how it was made, and "remix" it. In Scratch, the term "remixing" refers to the creation of any new version of a Scratch program by adding, removing or changing the programming blocks, images or sounds. In this section we use qualitative data from the Scratch website to provide social context for remixing and to suggest that credit plays an important role in how users conceive of appropriate remixing practice.

Remixing in Scratch is not only technically possible, it is something that the administrators of the website encourage and try to foster as a way for people to learn from others and collaborate. On every project page, the Scratch website displays a hyperlink with the text "Some rights reserved" that points to a child-friendly interpretation of the Creative Commons Attribution-Share Alike license under which all Scratch projects are licensed. ${ }^{1}$ Even the name Scratch is a reference to hip hop DJs' practice of mixing records. A large portion of all projects shared on the Scratch website $(28 \%)$ are remixes of other projects.

That said, remixing is not universally unproblematic in Scratch. Previous quantitative analysis of the the Scratch community showed that Scratch participants react both positively and negatively to the remixing of their projects and found that of those users who viewed a remix of their project, about one-fifth left positive comments while the same proportion of users accused the remixer of plagiarism [8]. This ambivalent reaction to remixing is echoed, and given additional texture, in the comments and complaints left by users on the Scratch website and sent to Scratch administrators.

For example, even before the Scratch website was publicly announced, a number of early adopters became upset when they found remixes of their projects on the website. Indeed, one of the very first complaints about Scratch occurred on the discussion forums where a 13 year-old boy asked:

Is it allowed if someone uses your game, changes the theme, then calls it 'their creation'? Because I created a game called "Paddling 1.5" and a few days later, a user called "julie" redid the background, and called it "her creation' and I am really annoyed with her for taking credit for MY project!! ${ }^{2}$

A similar complaint was sent to the website administrators by a 14-year old boy:

I think there should be a way to report plagiarized projects I've been seeing a lot of people's projects taken and renamed. This member, named kings651, has 44 projects, and most of them are made by other people. He even has one that I saw my friend make so I know he actually made it.

In other cases, the disagreements over remixing were more public and involved communication via projects and com-

\footnotetext{
${ }^{1}$ A copy of the current version of the kid-friendly license is available online at http://scratch.mit.edu/pages/license. The version available today encourages users to give credit manually in the project notes. A strong emphasis on credit-giving was added as a result of the findings reported here but was absent during the period of data collection for this study.

${ }^{2}$ All usernames and quotes from the website were changed to disguise the identities of participants.
} 
ments. For example, user koolkid15 wrote the following message in a comment which was left is response to a remix that shows a cat frowning:

Hi i'm koolkid15 the original creator of luigi disco jayman41 copied me!! and didn't even aknowladge me he didn't change anything !! I wrote or drew!! and jayman...if your reading this think about other people!!!!

Despite the fact that Scratch was conceived, designed, and launched as a platform for remixing, these users expressed their displeasure at remixing. That said, none of these users complained directly about the reuse of their project in general, but in terms of unfair "taking credit", plagiarism, and a lack of acknowledgment. Remixing was seen as problematic for koolkid15, for example, because of the nontransformative nature of reuse, the lack of acknowledgment of antecedent contributors, and the confusion about credit that would result.

Of course, other, more positive, scenarios around remixing also played out in Scratch. For example, jellogaliboo created a remix of Catham's project and wrote the following in the project notes: "i kinda copied Catham's "jetpackcat" game. i used the kitty, the blocks (i added and changed some), and the fuel thingy." Catham later posted his approval of the remix saying, "I like what you changed about my project!" Like this example, many of these positive experiences involved explicit credit-giving by a remixer to the creator of the antecedent project.

\section{DESIGN INTERVENTION: AUTOMATING ATTRIBUTION}

Several user complaints about remixing and plagiarism also included suggestions for how Scratch's designers might address them. For example, in response to the forum thread mentioned in the previous section, a 16 year-old proposed two potential design-based solutions:

Make it so you can only download a view of how your game/story/animation works. Or make it so downloadable Scratch files have read only protection. Maybe downloaded Scratch files, after being uploaded, are marked with the creators name at the bottom, and then any DIFFERENT people who edit it after are put on the list.

Influenced by these comments, Scratch administrators came to believe that negative responses towards remixing were often due to the fact that Scratch users did not acknowledge the sources of their remixes. As a result, these administrators implemented an architectural design change to the Scratch community along the lines suggested by the user in the second half of the quotation above.

The design change in question involved the introduction of a new technological facility that automatically identified and labeled remixes and inserted hyperlink pointers under each remix to the remix's antecedent and the antecedent's author (see Figure 3). Two days after the introduction of this feature, functionality was added to link to a comprehensive list

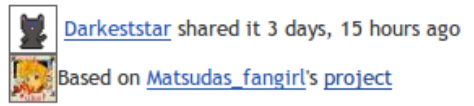

Figure 3. Example of the automatic attribution-giving statement displayed under remixes in the period after the design intervention.

stickylady shared it 1 year ago () (2) Some rights reserved

41 views, 7 remixes by 5 people, 3 downloads, in $\underline{2 \text { galleries }}$

Figure 4. Example of the link pointing to remixes of a project displayed under antecedent projects in the period after the design intervention.

of derivative works from the pages of antecedent projects (see Figure 4).

The new feature was announced in the discussion forums by an administrator of the website and user responses were positive. User terminator99 suggested that the change was, "Awesome." Another user, marsUp, posted a comment saying, "That's a very useful feature! I like that we can do pingpong like modding in Scratch." Users who did not visit the discussion forums also responded well to the the new feature. For example, user greekPlus posted a comment on a remix he created saying, " $\mathrm{i}$ remixed it for you but $\mathrm{i}$ do not know how to ad credit to you for thinking of it in the first place." A few minutes later he realized that the remix automatically displayed the attribution and posted the a comment saying, "never mind it did it for me. cool!"

\section{STUDY 1: HUMAN AND MACHINE ATTRIBUTION}

Although initial user feedback to the automatic attribution feature was positive, users continued to complain about remixing. In Study 1a, we present a quantitative analysis to more fully evaluate the effect of the technological design change described in the previous section. In Study 1b, we offer a parallel analysis of the relationship between manual crediting-giving by users and users' reactions to being remixed.

Both studies build on a dataset used in previous work by Hill, Monroy-Hernández, and Olson [8]. This dataset includes remix-pairs determined by an algorithm using detailed project metadata tracked by the Scratch online community. The dataset is limited in that it does not include projects whose concepts were copied by a user who had seen another's work but who did not actually copy code, graphics or sound. Similarly, the dataset contains no measure of the "originality" of projects or an indicator based on ideas that were taken from a source outside Scratch (e.g., a user may have created a Pacman clone which would not be considered a remix in our analysis).

The data presented here includes each coded reactions of the author of antecedent projects (i.e., originators) on remixes of their projects shared by other users in the site during a twelve week period after Scratch's launch from May 15 through October 28, 2007. Although 2,543 remixes were shared in this period, we limit our analysis to the 932 projects (37\% of 


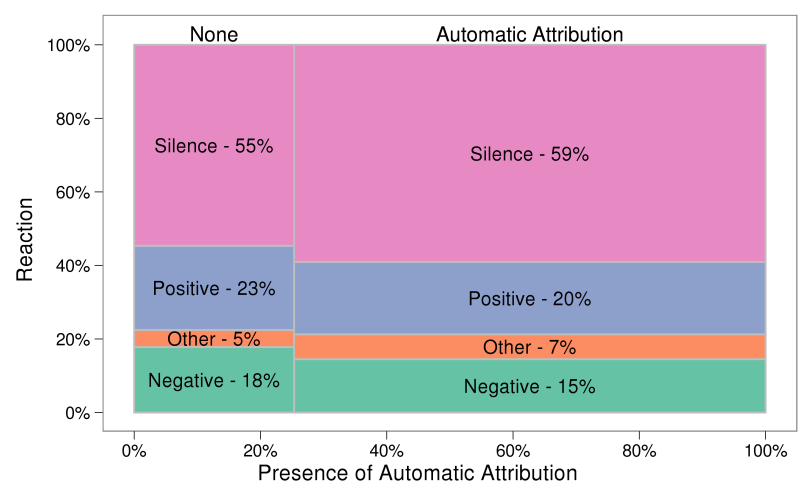

Figure 5. Mosaic plot showing the distribution of reactions of original creators who had viewed remixes of their project during the six week periods before and after the introduction of automatic attribution. The proportion of response types is shown along the y-axis. The proportion of projects viewed with, and without, automatic attribution is shown along the $\mathrm{x}$-axis. $(n=932)$

the total) that had been viewed at the time of data collection by the project originator - a necessary prerequisite to any response. Of these 932 remixes that were viewed by a project originator, 388 originators $(42 \%)$ left comments on the remixes in question. The remaining were coded as "silence." Comments left by originators were coded by two coders, blind to the hypotheses of the study and who were found to be reliable [8], as being positive, neutral, or negative. They were also coded as containing accusations of plagiarism (projects in which the the originator directly accused the remixer of copying, e.g., "Hello mr plagiarist", "Copycat!") or hinting plagiarism (projects in which the originator implied that the remixer had copied but did not state this explicitly, e.g., "I mostly pretty much made this whole entire game").

Unless it also contained an explicitly negative reaction, an accusation of plagiarism was not coded as "negative." However, because plagiarism tends to be viewed as negative within Scratch (as suggested by the quotations in the previous section) and more broadly in society [20], we re-coded accusations of plagiarism (both direct and hinting) as "negative" except, as was the case in several comments coded as "hinting plagiarism," when these accusations were in comments that were also coded as positive. Previous published work using this dataset, and subsequent robustness checks, show that our results are substantively unchanged if we exclude these explicit charges of plagiarism from the "negative" category or exclude only the weaker "hinting plagiarism" accusations.

\section{Study 1a: Automatic Attribution}

To test the effectiveness of automatic attribution, we consider the effect of the design intervention described in the previous section. The design change took place six weeks after the public launch of the Scratch community and at the precise midpoint in our data collection window. The intervention affected all projects hosted on the Scratch online community including projects shared before the automatic attribution functionality was activated. As a result, we classify originators' reactions as occurring outside a technological regime of automatic attribution when a project was both uploaded and viewed by a project's originator before automatic attribution functionality was activated.

A comparison of the distribution of coded comments between positive, neutral, negative, and silent in the periods before and after the intervention suggests that the introduction of automatic attribution had little effect on the distribution of reaction types (See Figure 5). Although the period after the intervention saw a larger proportion of users remaining silent and a smaller proportion of both positive and negative comments, $\chi^{2}$ tests suggest that there is no statistically significant difference in originator reactions between remixes viewed before or after the introduction of automatic attribution $\left(\chi^{2}=3.94 ; d f=3 ; p=0.27\right)$. As a result, we cannot conclude that the there is any relationship between the presence, or absence, of an automatic attribution system in Scratch and the distribution of different types of reactions.

These results suggest that automatic attribution systems may have limited effectiveness in communities like Scratch. Of course, our analysis is not without important limitations. For example, the existence of an automatic attribution regime may also affect the behavior of users preparing remixes. A remixer might avoid making perfect copies of projects if they know that their copies will be attributed and are more likely to be discovered.

\section{Study 1b: Manual Crediting}

While the introduction of an automatic attribution feature to Scratch appears to have had a limited effect on originators responses to remixes of their projects, the presence or absence of credit was a recurring theme in discussions on Scratch online forums - as shown in the quotes in the previous section - and in many of the coded reactions from the periods both before and after the introduction of automatic attribution. Indeed, in project descriptions or notes from the periods both before and after the change, remixers frequently "manually" gave credit to the originators of their work. Even after remixes were automatically attributed to originators, remixers who did not also give credit manually - essentially producing information redundant to what was already being displayed by the system - were criticized.

For example, after the introduction of automatic attribution functionality, a user left the following comment on a remix of their project:

Bryan, you need to give me Pumaboy credit for this wonderful game that I mostly pretty much kinda totally made this whole entire game ... and that you need to give me some credit for it

For this user, automatic attribution by the system did not represent a sufficient or valid form of credit-giving. In the following study, we test for this effect of "manual" creditgiving by remixers on coded response types using a method 


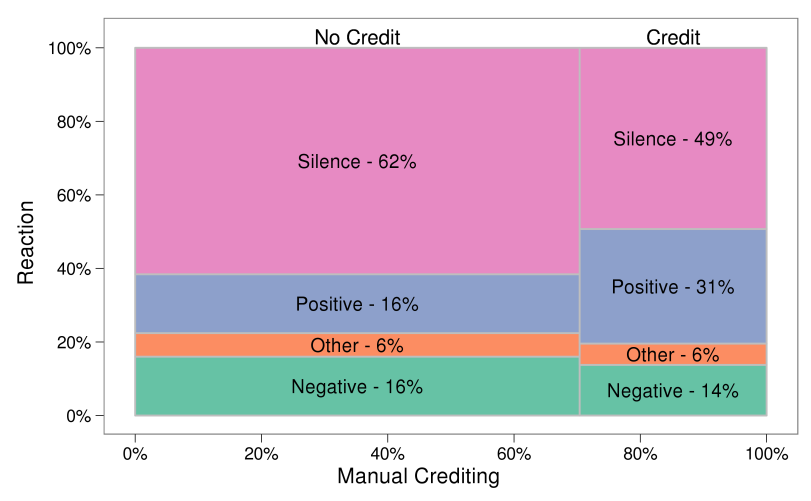

Figure 6. Mosaic plot showing the distribution of reactions of original creators who had viewed remixes of their project and indicating whether they left manual credit. The proportion of response types is shown along the $y$-axis. The proportion of projects including and omitting manual credit is shown along the $\mathbf{x}$-axis. $(n=932)$

that parallels the analysis in Study 1a and that uses the same dataset.

Manual crediting can happen in multiple ways. Exploratory coding of 133 randomly projects showed that $35(36 \%)$ of each remix pair gave credit. Of these 35 projects, 34 gave credit in the project description field while 1 project only gave credit in a "credits" screen inside the game. As a result, the authors of this study split the sample of projects used in the Study 1a and coded each of of the user-created descriptions for the presence or absence of explicit or manual credit-giving.

To first establish that we are examining distinct behaviors, we attempted to establish that automatic and manual attribution do not act as substitutes for each other. As suggested by our qualitative findings and our results in Study 1a, we found little difference in the rate of explicit credit giving between projects created in the presence or absence of automatic attribution. Overall, 276 (about 30\%) of the 932 projects in our sample offered explicit credit in the description field of the project. Manual crediting-giving was a widespread practice both before automatic attribution, when $31 \%$ of projects in our sample offered explicit credit, and after, when $27 \%$ did so. The difference between these two periods was not statistically significant $\left(\chi^{2}=1.41 ; d f=1 ; p=0.24\right)$. Previous work studying Jumpcut, a video remixing website, supports the idea that automatic and manual credit giving are not interchangable phenomena. One Jumpcut user with permission to creative derivative works commented that they, "still feel a moral obligation to people as creators who have a moral right to be attributed (and notified) despite the physical design which accomplishes this automatically" [5].

We measured effectiveness of manual credit giving using a parallel analysis to Study 1a. As in Study 1a, we compared the distribution of originator reactions in the presence, and absence, of manual credit-giving by remixers. We found that negative reactions are less common in the presence of manual credit but that this difference is very small (from $16 \%$ without manual credit to $14 \%$ with it). However, we see that the proportion of users who react positively almost doubles in the presence of credit-giving (from $16 \%$ with no crediting to $31 \%$ in its presence). A graph of these results are shown in Figure 6. Tests show that we can confidently reject the null hypothesis that these differences in the distribution of reactions are due to random variation $\left(\chi^{2}=27.60 ; d f=3 ; p<0.001\right)$.

Also important to note is a difference in the number of users who are silent after viewing a project $(62 \%$ in the absence of manual credit versus $49 \%$ in its presence). This larger proportion of commenting in general may have an important substantive effect on the discourse and behavior on the site because silent originators may, for obvious reasons, have a more limited effect on attitudes toward remixing and user experience than vocal users do. As a robustness check, we considered the reaction of only originators who left comments $(n=388)$ and found that even with a smaller sample, our result were stronger. In the restricted sample, $41 \%$ reacted negatively when they were not given credit. However, only $27 \%$ did so when they were credited. Similarly, $42 \%$ of users who left comments on projects that did not give credit manually left positive messages. Nearly two thirds of comments $(61 \%)$ were positive when credit was given. These differences, in the reduced sample that includes only explicit reactions, were also statistically significantly different $\left(\chi^{2}=14.09 ; d f=2 ; p<0.001\right)$. We include the large number of silent participants because we believe that nonresponse is an important type of reaction with real effects on the community. Understanding the reasons behind nonresponse and the effect of silence in response to different types of credit giving remains an opportunity for further research.

Although not presented here due to limited space, we followed the general model of previous work using this dataset [8] and tested logistic regression models on dichotomous variables indicating the presence of negative and positive reactions and found that basic relationships described above were robust to the introduction of a control for the the intervention, to an interaction between these two variables, and to controls for the gender and age of originators and to the antecedent project's complexity. Both before or after the intervention, manual crediting resulted in more positive comments by the originators of remixed projects. Of course, the results presented here are uncontrolled, bivariate, relationships and we caution that these results, while provocative, should still be viewed as largely tentative. As we show in the subsequent qualitative analysis, attribution and credit-giving are complex social processes. We do not claim that the preceding analyses capture it fully.

\section{STUDY 2: INTERVIEWS WITH PARTICIPANTS}

In order to explore the reasoning behind young people's remixing behavior and attitudes toward attribution as we observed it in Study 1, we engaged in a second qualitative study and directly asked kids what role attribution and credit plays in their moral evaluations of remixing. 


\begin{tabular}{|c|c|c|c|}
\hline Name (pseudonym) & Age & Gender & Relationship to Scratch \\
\hline Nicole & 10 & $\mathrm{~F}$ & She has created with hundreds of Scratch projects, mainly animations and art ones. \\
\hline Kyle & 14 & M & Casual user of Scratch, interested in math/science simulations and video games. \\
\hline Amy & 15 & $\mathrm{~F}$ & Avid photographer, has never used Scratch. \\
\hline Charles & 9 & $\mathrm{M}$ & $\begin{array}{l}\text { Active member of a subgroup of Scratch interested in simulation of operating } \\
\text { systems. }\end{array}$ \\
\hline Ryan & 12 & $\mathrm{~F}$ & Long-time member of the Scratch community. Creates complex video games. \\
\hline Jon & 9 & M & Casual user of Scratch, collaborates with Scratch friends in person. \\
\hline Jake & 11 & M & Casual user, likes making video games. \\
\hline Cody & 16 & M & Creates hip hop accessories, not active in Scratch. \\
\hline Paul & 9 & M & Creates Scratch projects with a focus on engineering and video games. \\
\hline Jimena & 17 & $\mathrm{~F}$ & $\begin{array}{l}\text { Highly technical teen with programming experience but no experience with } \\
\text { Scratch. }\end{array}$ \\
\hline Madeline & 14 & $\mathrm{~F}$ & Very popular animator in the Scratch community. \\
\hline Susie & 10 & $\mathrm{~F}$ & $\begin{array}{l}\text { Has created hundreds of projects including games, animations and art, but prefer- } \\
\text { ring art. }\end{array}$ \\
\hline
\end{tabular}

Table 1. Table listing details of interviewees used in Study 2. $(n=12)$

\section{Methodology}

We conducted twelve one-hour semi-structured interviews with kids aged 8 to 17 years old. All of the interviewees had experience using computers and had access to the Internet at home. All the interviewees live in the United States except for one who lives in New Zealand. The participants were recruited via the Scratch website and during meet-ups with educators, teachers and young Scratch users. Eight of the interviews were conducted in person, in the Boston area, and the rest over the phone or voice over IP. The interviews were audio-recorded and transcribed before fully analyzing them. Nine of the interviewees were members of the Scratch community. The remaining three did not use Scratch but were included as a way to check if people who do not use Scratch have similar views about remixing, attribution, and credit. We found no substantive difference between the Scratch users and non-users in their answers to questions related to the hypothetical automatic and manual mechanism for attribution.

Before each interview, subjects completed a survey that elicited demographic information and posed questions about their familiarity with other technologies and which was primarily designed to get a sense of the interviewees' social and technical background. Interviews were structured around a protocol that included a set of nine fictional remixing cases intended to elicit conversations about remixing. ${ }^{3}$ The cases were inspired by Sinnreich et al.'s theoretical work and from three years of experience moderating the Scratch community. They were designed to present cases where remixing could be controversial but where there is no clear "correct" answer. The goal of the cases was to offer a concrete, and common, set of dilemmas to stimulate broad conversations about attitudes toward remixing.

The cases were presented in the form of printed screenshots of different project pages from the Scratch website (anonymized to avoid referring to real cases that users might

\footnotetext{
${ }^{3}$ Our interview protocol including example cases is available at http://www.media.mit.edu/ andresmh/chi2011/interview.html.
}

have seen). The print outs were shown to the interviewees (or discussed over the phone) while explaining each case. All the cases included a remix and its corresponding antecedent project. The cases varied in the presence of automatic attribution, manual credit, and the degree of similarity between the remix and its antecedent. For example, the first three cases were:

1. A remix and its antecedent are identical. The project notes only describe how to play the video game. The remix shows the automatic attribution but no manual credit on the notes.

2. A remix and its antecedent are different (as seen visually and in project metadata) but one can clearly see the influence of its antecedent project. The project notes of the remix show manual credit but no automatic attribution. The interviewee was told to imagine the site had a glitch that prevented it from connecting it to its antecedent.

3. The same set of remix and antecedent projects as in (2) but this time automatic attribution is displayed but manual credit is not.

Each of the interview logs was coded using inductive codes and grounded theory [3]. The coded responses were analyzed based on categories related to how interviewees answered specific questions about the distinction between automatic attribution and manual credit.

\section{Results}

Confirming the results of Study 1, for users of Scratch, automatic attribution was generally seen as insincere and insufficient. Throughout the interviews, we found that for most of the kids, getting explicit credit from another person was preferred over attribution given automatically by the system. When asked why, kids often responded that knowing that another person had cared enough to give credit was valued more than what the computer system would do on its own. The fact that it takes some work, albeit minimal, to write an acknowledgment statement, sends a signal of empathy, authenticity and good intentions [6]. Amy articulated this 
when explaining why she preferred getting credit from another person:

I would like it even more if the person did it [gave credit] on their own accord, because it would mean that $[\ldots]$ they weren't trying to copy it, pirate it.

Similarly, Jon explained, "No [the "Based on" is not enough], because he [the remixer] didn't put that, it always says that." For Jon, automatic attribution is not authentic because it is always there and, as a result, it is clear that is not coming from the person doing the remix.

Most of the interviewees seemed to have a clear notion of what they think a moral remix should be. For some, it is all about making something different. Jake for example, defines a "good" remix as, "if it has a bunch of differences then it's a good remix. If it has like two, then it's bad." In addition to the differences between the remix and its antecedent project, for some, manual credit is part of what makes it moral. Charles said, "[remixing] is taking somebody else's project and then changing a lot of it and sharing it and giving credit." Continuing, Charles explained:

If Green had actually said in the project notes, "This is a remix of Red's project, full credit goes to him," then I would consider it a remix. But this [pointing at a remix without manual credit] is definitely a copy.

Likewise, Ryan mentions that a fictional remix was, "perfectly fine because they gave credit in the project notes."

Interviewees suggested that manual credit also allows users to be more expressive. For example, Susie explained that expressiveness is the reason that she prefers manual credit through the project notes saying, "I think the manual one is better because you can say 'thank you' and things like that. The automatic one just says 'it's based on."' Susie also notes that for her, the project notes are a space where a creator can express her wishes in regards to her intellectual property, independent, and even in contradiction to, the license of the projects:

If I do a project that has music that I really like, I often download the project, take the music. Unless it says in the project notes, "Do not take the music."

For Susie and other users of Scratch, the project notes are a space for more than just instructions on how to interact with one's project; they are an expressive space where one can communicate with an audience without having to encumber the creative piece of work with it.

Others point at the fact that people do not pay as much attention to automatic attribution statement as much they do to the manual credit left in project descriptions. Jake, for example, explains that, while he agrees there is some usefulness to having both, project notes still are more important, "because, you know, sometimes people just like skim through a project and you don't see it 'til the end." Jake continued to say that creators that do not have both should get a "warning."
Even though interviewees value manual credit, they still see the usefulness of the automatic mechanism as some sort of community-building prosthetic device - an explanation for the positive reactions to the feature's initial introduction. For example, Nicole argues that while manual credit on the notes has more value for her, the automatic attribution is useful as a backup and because it provides a link:

Well, I think that they should probably write in the notes that - then it should also say "Based on blank's project," just in case they forget, and also because it gives a link to the original project and it gives a link to the user so you don't have to search for it.

A similar explanation was articulated on a comment exchange on one the website's galleries. A teenage girl that actively participates in Scratch explained the pragmatic value of automatic attribution saying, "the 'based on' thingy, it gives a link, and we all luv links, less typing," before reiterating that manual credit is more valuable:

at the beginning i thought that you don't have to give credit when the "based on" thingy is in there, but i realized a lot of people don't look at that, and i noticed people confused the remix with the original.

Creating a Scratch project is a complicated task. A project's sources can be diverse and the creator can easily forget to acknowledge some, as Paul explains, when asked to choose between a system of manual credit or automatic attribution:

The thing is, it would be a lot better if they had both. Because, sometimes people probably just forget to do that. And then people would not know.

There are also situations where interviewees recognize what Posner calls the "awkwardness of acknowledgment," that is, situations where credit is not really needed and it can be an unnecessary burden or go against the aesthetics of the work [20]. For example, Paul mentioned that sometimes, there are some projects in Scratch that are remixed so much - like the sample projects that come with Scratch or some "remix chains" ${ }^{4}$ - where credit is not necessary:

There's this one called "perfect platformer base" which a lot of people remix. So I don't think that needs any credit. It's not actually a real game. It's all the levels and stuff are just demonstrations.

Since manual crediting has a higher emotional value, some kids mentioned that conflicts over remixing could be addressed by the administrators of the site by editing the project of the remix in question, as a way to enforce credit without transforming it into attribution. Doing so would make it appear that a remixer had credited an antecedent when they had not. Susie offers a suggestion along these lines when asked about how the administrators of the website should deal with a case of a complaint over a remix

\footnotetext{
${ }^{4}$ Remix chains typically start with someone sharing a project inviting others to remix (i.e. "add your animated avatar to the park.")
} 
that is a parody of someone else's project. Susie suggested that, "I might remove the project but I might not, you know, maybe I would edit the notes to to give credit." Similarly, Charles described his approach for solving conflicts if he was the administrator of the website suggesting that, "I probably just would stay out of the argument. I probably wouldn't remove it [the remix], I'd just add something in the project notes [like] 'based on Gray's project."'

This phenomena of giving less value to technologically simplified social signals is experienced in other social platforms. For example, Amy expressed how on the social network site Facebook, she loves to get comments on her photographs but dislikes those who do not leave comments or opt instead to press the "I like it" button:

I love when people comment on my pictures. Everybody sees them, because they tell me they have. I'm like, "Oh really? That's great. Why didn't you comment?" I don't like it when people just "like it", because you know they have something to say about it; they just don't. It's like, if they like it, then [they should] take the time to say something.

Although not designed to be a random sample, these interviews support the proposition that both Scratch participants and other young people share a set of norms about characteristics that determine what a "good" or moral remix is. Among these norms, acknowledging one's sources seems to play a central role. However, participants also seem to share the opinion that this norm is not satisfied through an automated process. They clearly understand the pragmatic value of automating acknowledgment-giving, but they do not see it as a substitute for adherence to the social norm of creditgiving. They also see it as void of emotion and expressiveness. For Scratch users, normative constraints are separate from architectural constraints and one cannot replace the other. These findings support and enrich the results from our first study and help us understand better how Scratch participants, and perhaps kids in general, experience authorship norms and automation in online spaces.

\section{CONCLUSIONS}

Our results from Study 1a called into the question the effectiveness of automatic attribution functionality in encouraging more positive user reactions in Scratch. We build on these results in Study $1 \mathrm{~b}$ to suggest that manual crediting may do the work that Scratch's designers had hoped automatic attribution would. Results from the analysis of user interviews presented in Study 2 help to answer the question of "why?" and suggest that users find manual credit to be more authentic and more meaningful to users because it takes more time and effort. Usually, UI improvements are designed to help reduce the time and effort involved in using a system. But in trying to help users by attributing automatically, Scratch's designers misunderstood the way that attribution as a social mechanism worked for Scratch's users. Our fundamental insight is that while both attribution and credit may be important, they are distinct concepts and that credit is, socially, worth more. A system can attribute the work of a user but credit, which is seen as much more important by users and which has a greater effect on user behavior, cannot be done automatically. Computers can attribute. Crediting, however, takes a human.

As we suggested in our introduction, this fundamental result leads to two distinct contributions. First, and more specifically, our analysis offers an improved understanding of the way that attribution and credit works in user-generated content communities over what has been available in previous work. Our two studies suggest that scholars are correct to argue that credit plays an important role in social media communities and offer empirical confirmation for the important role that authenticity plays in how users conceptualize credit. In our in-depth interviews, we explain some of the reasons why this may be the case. Second, through our evaluation of an unsuccessful technological design, our work offers a broader, if more preliminary, contribution in suggesting an important limit of designers' ability to support community norms in social media systems. As the literature on design and social media grows, the importance of good support for communities with healthy norms promoting positive interactions is likely to increase. In attempting to design for these norms, we suspect that researchers will increasingly encounter similar challenges.

We argue that designers should approach interventions iteratively. This design approach can be understood through the theoretical lens of the social construction of technology [19]: designers can't control technological outcomes which must be built through a close relationship between designers and users. Designers must move away from seeing their profession as providing solutions. They must channel users, work closely with them, and iterate together, to negotiate and achieve a set of shared goals.

The prevalence of user-generated content sites stresses the importance of how online social spaces should deal with issues of attribution and our results are likely to be immediately relevant to designers. For example, the Semantic Clipboard is a tool built as a system of automatic attribution for content reuse [22]. Developed by researchers who found a high degree of Creative Commons license violations around the re-use of Flickr images, the tool is a Firefox plugin that provides, "license awareness of Web media," and enables people to automatically, "copy [media] along with the appropriate license metadata." Our results suggest one way that this approach may fall short.

However, automatic attribution is not the only way that technologists can design to acknowledge others' contributions. Indeed, our results suggest that there may be gains from design changes which encourage credit-giving without simply automating attribution. For example, Scratch's designers might present users with a metadata field that prompts users to credit others and suggests antecedent authors whose work the system has determined may have played a role. This affordance might remind users to credit others, and might increase the amount of crediting, while maintaining a human role in the process and the extra effort that, our research has 
suggested, imbues manual credit giving with its value. We suggest that in other social media communities, similar affordances that help prompt or remind users to do things that a system might do automatically represent a class of increasingly important design patterns and a template for successful design interventions in support of community norms.

\section{ACKNOWLEDGMENTS}

This research was supported by Microsoft Research. Scratch is a project of the Lifelong Kindergarten Group at the MIT Media Lab with financial support from the National Science Foundation award ITR-0325828, Microsoft Corp., Intel Foundation, Google, the MacArthur Foundation and the MIT Media Lab research consortia.

\section{REFERENCES}

1. Benkler, Y. The Wealth of Networks: How Social Production Transforms Markets and Freedom. Yale University Press, Oct. 2007.

2. Brown, G. O. Announcing (and explaining) our new 2.0 licenses. http://creativecommons.org/weblog /entry/4216, May 2004.

3. Charmaz, K. Constructing Grounded Theory: A Practical Guide Through Qualitative Analysis. Sage Publications, London, 2006.

4. Cheliotis, G., and Yew, J. An analysis of the social structure of remix culture. In Proc. C\&T 2009 (University Park, PA, USA, 2009), ACM, 165-174.

5. Diakopoulos, N., Luther, K., Medynskiy, Y. E., and Essa, I. The evolution of authorship in a remix society. In Proc. HT 2007 (Manchester, UK, 2007), ACM, 133-136.

6. Donath, J. Signals in social supernets. Journal of Computer-Mediated Communication 13, 1 (2008), 231-251.

7. Friedman, B. Social judgments and technological innovation: Adolescents' understanding of property, privacy, and electronic information. Computers in Human Behavior 13, 3 (Aug. 1997), 327-351.

8. Hill, B. M. H., Monroy-Hernández, A., and Olson, K. Responses to remixing on a social media sharing website. In Proc. ICWSM 2010 (Washington, D.C., 2010), AAAI, 74-81.

9. Ito, M., Okabe, D., and Matsuda, M. Personal, Portable, Pedestrian: Mobile Phones in Japanese Life. The MIT Press, 2006.

10. Jenkins, H. Convergence Culture: Where Old and New Media Collide. New York University Press, New York, 2006.

11. Lessig, L. Code: And Other Laws of Cyberspace, Version 2.0. Basic Books, Dec. 2006.

12. Lessig, L. Remix: Making Art and Commerce Thrive in the Hybrid Economy. Penguin Press HC, The, Oct. 2008.
13. Luther, K., Diakopoulos, N., and Bruckman, A. Edits \& credits: exploring integration and attribution in online creative collaboration. In Proc. CHI 2010 (Atlanta, Georgia, USA, 2010), ACM, 2823-2832.

14. Manovich, L. Remix and remixability. http://www.manovich.net/DOCS/Remix_modular.doc, 2005.

15. Monroy-Hernández, A., and Resnick, M. Empowering kids to create and share programmable media. interactions 15, 2 (2008), 50-53.

16. Olson, K. R., and Shaw, A. 'No fair, copycat!': what children's response to plagiarism tells us about their understanding of ideas. Developmental Science (2010).

17. Palfrey, J., and Gasser, U. Born Digital: Understanding the First Generation of Digital Natives. Basic Books, Aug. 2008.

18. Perkel, D. Copy and paste literacy: Literacy practices in the production of a MySpace profile. Informal Learning and Digital Media (2006).

19. Pinch, T. J., and Bijker, W. E. The social construction of facts and artefacts: Or how the sociology of science and the sociology of technology might benefit each other. Social Studies of Science 14, 3 (Aug. 1984), 399-441.

20. Posner, R. A. The little book of plagiarism. Pantheon Books, Jan. 2007.

21. Resnick, M., Maloney, J., Monroy-Hernández, A., Rusk, N., Eastmond, E., Brennan, K., Millner, A., Rosenbaum, E., Silver, J., Silverman, B., and Kafai, Y. Scratch: programming for all. Commun. ACM 52, 11 (2009), 60-67.

22. Seneviratne, O., Kagal, L., and Berners-Lee, T. Policy-Aware content reuse on the web. In The Semantic Web - ISWC 2009. 2009, 553-568.

23. Seneviratne, O., and Monroy-Hernández, A. Remix culture on the web: A survey of content reuse on different User-Generated content websites. In Web Science (Raleigh, NC, Apr. 2010).

24. Shaw, R., and Schmitz, P. Community annotation and remix: a research platform and pilot deployment. In Proc. MM 2006 (Santa Barbara, California, USA, 2006), ACM, 89-98.

25. Sinnreich, A., Latonero, M., and Gluck, M. Ethics reconfigured. Information, Communication \& Society 12, 8 (2009), 1242.

26. Thom-Santelli, J., Cosley, D. R., and Gay, G. What's mine is mine: territoriality in collaborative authoring. In Proc. CHI 2009 (Boston, MA, USA, 2009), ACM, 1481-1484. 